\title{
COMUNIDADE DE PRÁTICA E CUIDADO EM SAÚDE MENTAL: UMA REVISÃO SISTEMÁTICA
}

\author{
COMMUNITY OF PRACTICE AND MENTAL HEALTH CARE: \\ A SYSTEMATIC REVISION
}

\author{
COMUNIDAD DE PRÁCTICA Y CUIDADO EN SALUD MENTAL: \\ UNA REVISIÓN SISTEMÁTICA
}

\author{
Taís Quevedo Marcolino ${ }^{1}$ \\ Eliane Nascimento Fantinatti ${ }^{2}$ \\ Alana de Paiva Nogueira Fornereto Gozzi ${ }^{3}$
}

Resumo O cuidado em saúde mental é dependente de recursos humanos qualificados para responder dinamicamente às necessidades dos usuários dos serviços. A qualificação desse cuidado pela reflexão sobre os processos de trabalho é essencial para a efetivação da mudança para uma perspectiva comunitária e biopsicossocial. A comunidade de prática é um referencial teórico-metodológico que fomenta a aprendizagem colaborativa por meio do engajamento mútuo em projeto comum e da negociação de significados. A pesquisa teve por objetivo sistematizar a utilização da comunidade de prática para melhoria do cuidado em saúde mental via revisão sistemática no Portal de Periódicos da Capes e na Biblioteca Virtual em Saúde, em março de 2015. De 19 artigos encontrados, nove foram selecionados para análise. Os estudos avaliaram positivamente a comunidade de prática para melhorar os serviços oferecidos à população, oferecer capacitação com especialistas e implementar novos métodos de trabalho. Destacaramse fragilidades (recursos financeiros, desalinhamento inicial entre os participantes, conflitos grupais) e potencialidades (engajamento real dos participantes; participação virtual; acesso a especialistas; integração de profissionais, usuários e familiares num único projeto; necessidades formativas em contexto). Espera-se elucidar possibilidades para a criação de estratégias formativas e investigativas para educação permanente em saúde mental.

Palavras-chave saúde mental; educação permanente; comunidade de prática; recursos humanos em saúde; prática profissional.
Abstract Mental health care is dependent on qualified human resources to respond dynamically to the service users' needs. This qualification by reflection on the work processes is essential for effective change to a community and biopsychosocial perspective. The Community of Practice is a theoretical framework that fosters collaborative learning through mutual engagement in common projects and negotiation of meanings. The research had as objective to systematize the use of Community of Practice for improving mental health care, by systematic review at Capes Portal and Virtual Health Library in March 2015. We found nineteen articles and nine of them were selected for analysis. The studies evaluated the Community of Practice as positive to improve the services offered to the population, provide training from experts, and implement new methods of work. They highlight weaknesses (financial resources, initial misalignment between the participants, group conflicts) and potentialities (real engagement of participants, virtual participation, access to specialists, integration of professionals, users and families in a single project; training needs in context). We expected to clarify possibilities for training and investigative strategies for continuing education in mental health. Keywords mental health; continuing education; community of practice; health mainpower; professional practice. 


\section{Introdução}

Mesmo a atenção em saúde mental considerada um direito fundamental, ainda é um setor parcamente financiado, com impactos no acesso da população ao cuidado efetivo e humanizado, além da discriminação, isolamento e desconhecimento das reais necessidades dos indivíduos que sofrem de um transtorno mental, inclusive por parte de profissionais da área (Organização Mundial da Saúde, 2005).

Thornicroft e Tansella (2010) ressaltam o papel central que o fator humano ocupa nesse cuidado (meta primária), dando destaque à importância do entendimento e atendimento das necessidades da equipe multidisciplinar (meta secundária): “Os serviços de saúde mental dependem muito mais de recursos humanos do que de equipamentos tecnológicos" (Thornicroft e Tansella, 2010, p. 149).

O deslocamento da perspectiva institucional-hospitalar para a perspectiva comunitária não está pautado apenas na realocação física dos locais de tratamento, mas também na reorientação do processo de trabalho, demandando dos profissionais uma orientação biopsicossocial eclética que os torne capazes de considerar os usuários em seu contexto territorial. Além disso, é premente pautar suas intervenções nas necessidades do indivíduo e nas de sua família e demais membros da rede social de suporte, independentemente de seu núcleo de conhecimento e formação profissional. Nesse contexto, a boa prática no cuidado depende da flexibilidade de cada profissional em responder de maneira dinâmica às necessidades dos usuários, que se modificam constantemente (Onocko-Campos et al., 2009; Thornicroft e Tansella, 2010).

A mudança do cuidado institucional para o cuidado territorial não deveria ser motivo para redução do orçamento destinado à saúde mental, embora essa seja uma realidade em diversos países.

A questão do financiamento neste campo ainda é de ordem bastante precária. Ainda que as doenças mentais contribuam com $12 \%$ da carga global de doenças, apenas $2 \%$ de todo o gasto mundial com o cuidado em saúde é destinado ao cuidado em saúde mental (Thornicroft e Tansella, 2010, p. 119).

Esse fato compromete não apenas o acesso das pessoas com transtornos mentais ao cuidado, mas também a implementação de desenvolvimento profissional contínuo, em especial para assistência comunitária em saúde mental. A sustentação dessa perspectiva de cuidado envolve a construção e a implementação de equipamentos que respondam às demandas e, principalmente, a exigência de novas práticas profissionais e da nova organização do trabalho na saúde mental, que impliquem os trabalhadores com seu processo de trabalho - de modo a construir novas formas de cuidado e um novo modo de 
significar o trabalho em saúde, em uma proposta educacional que alie aspectos cognitivos e subjetivos (Franco, 2007; Lima e Ghirardi, 2008).

Assim, processos de educação continuada, permanente ou educação situada ou em serviço têm papel fundamental para a construção de boas práticas em saúde mental, pois possibilitam o engajamento dos profissionais em constante reflexão sobre a prática e a (res)significação de suas experiências cotidianas, além do compartilhamento de informações que venham a se constituir norteadoras da prática. Nesse sentido, a educação permanente traz alguns aportes importantes nessa perspectiva de produção de conhecimento para a transformação de práticas, pois centra-se em processos de mudança a partir do cotidiano vivido pelos profissionais em construção conjunta. Desse modo, os possíveis problemas e dilemas demandam ser explicitados pelos trabalhadores, que, considerando a complexidade e a particularidade das situações, precisam se afastar de soluções causa-efeito para construir soluções que envolvam diferentes perspectivas, sendo levados a movimentos intensos e dinâmicos que exigem da equipe modificações e aprendizados constantes (Brasil, 2014, 2007; Davini, 1995; Flores, Oliveira e Zoche, 2016; Macedo, Albuquerque e Medeiros, 2014; Castro e Campos, 2014).

Davini (1995), importante pesquisadora da área, cita algumas razões para a implementação de processos e programas de educação permanente, como implantação de novos programas e ações inovadoras pelos serviços de saúde; novas demandas da comunidade para os serviços; reorientação das políticas sanitárias; mudanças previsíveis a curto e médio prazos, nas condições de saúde, baseadas em projeções epidemiológicas. Para Macedo, Albuquerque e Medeiros (2014), a mudança esperada pelas normativas ligadas à educação permanente na saúde brasileira (Brasil, 2014, 2007) ainda não mostrou reflexos na formação profissional, pautando os desafios do monitoramento e da avaliação de práticas formativas, assim como da constante invenção de práticas educacionais que busquem a transformação dos 'recursos humanos' em profissionais capazes de refletir sobre seus processos de trabalho, na complexidade do Sistema Único de Saúde (SUS).

Nessa direção, a comunidade de prática e identidade (CoP), de Ethiènne Wenger (1998), tem sido um referencial teórico-metodológico bastante utilizado para promover e investigar desenvolvimento e prática profissional (Cubas et al., 2015; Galheigo et al., 2017; Ramos e Manrique, 2015; Welch e Dawson, 2006), pois organiza-se pela participação ativa de pessoas em um projeto comum, centrado na negociação de significados. Esse referencial sustenta-se no pressuposto de que compartilhar e produzir diferentes tipos de repertório que construam e moldem o conhecimento a todo momento encoraja os participantes a refletirem, pesquisarem, analisarem e avaliarem coletivamente suas próprias ações, valores e conhecimentos. Esse processo é dinâmico, pois novos conhecimentos podem ser gerados no decorrer dele, desde que os pro- 
jetos favoreçam a interação entre diferentes elementos estruturadores (como propostas de trabalho preestabelecidas) e a habilidade de negociar novos direcionamentos, que emergem do contexto inerente ao grupo (Wenger, 1998).

Desse modo, ao se levar em consideração a potencialidade do referencial da comunidade de prática para fomentar processos formativos em saúde mental, realizamos uma revisão de literatura para buscar compreender como esse referencial vem sendo utilizado em contextos de prática relacionados ao campo da saúde mental, qual seu impacto e efetividade, bem como quais são os aspectos facilitadores e os dificultadores da aprendizagem nesse processo.

\section{Metodologia}

Realizou-se uma revisão sistemática de literatura, forma de pesquisa que utiliza informações obtidas em publicações científicas acerca de um tema de interesse. Esse tipo de pesquisa depende de uma busca sistematizada, seguida de apreciação crítica e síntese das informações obtidas, sendo capaz de oferecer ao leitor um resumo das evidências relacionadas a uma estratégia de intervenção específica (Linde e Willich, 2003).

A busca foi realizada em março de 2015 no Portal de Periódicos da Coordenação de Aperfeiçoamento de Pessoal de Nível Superior (Capes) (<http:// www.periodicos.capes.gov.br/>), que congrega diversas bases de dados brasileiras e internacionais com mais de 37 mil publicações periódicas, e na Biblioteca Virtual em Saúde (BVS) (<http://www.bireme.br/php/index.php>), sem delimitação de período, nas línguas portuguesa e inglesa, com os descritores comunidade de prática/community of practice e saúde mental/mental health. Os critérios de inclusão dos artigos foram: serem pesquisas ou relatos de experiência que descrevessem o uso da comunidade de prática no campo da saúde mental; estarem publicados nas línguas portuguesa ou inglesa. Para seleção dos artigos, utilizou-se a leitura do título, de palavras-chave e resumos dos artigos. Em caso de dúvidas, realizou-se a leitura completa dos artigos.

Todos os dados foram incluídos no software StArt (State of the Art through Systematic Review), ferramenta computacional de apoio a revisões sistemáticas que oferece suporte às etapas de planejamento, execução e análise final de dados (Fabbri et al., 2016).

Os dados selecionados para a análise foram: população-alvo do estudo; tipo de pesquisa (qualitativa, quantitativa ou mista); período de publicação; formato em que a comunidade de prática se desenvolveu (virtual, presencial ou ambos); formação dos autores; área do periódico na qual o artigo foi publicado; país de publicação; objetivos da comunidade de prática; tempo de duração; número de participantes. Além disso, utilizou-se análise temática (Bardin, 2011) para identificar núcleos de sentido, similaridades e diferenças, de modo a prover análise crítica dos resultados. 
A pesquisa "Comunidade de prática em Terapia Ocupacional: impacto da aprendizagem colaborativa para o cuidado em saúde mental", que originou este artigo, foi fruto de iniciação científica da primeira autora, realizada na Universidade Federal de São Carlos, com a orientação da terceira autora, tendo obtido financiamento do Programa Institucional de Bolsas de Iniciação Científica/Conselho Nacional de Desenvolvimento Científico e Tecnológico (Pibic/CNPq).

\section{Resultados e discussão}

No Portal de Periódicos da Capes foram encontrados 19 artigos, sendo que oito satisfaziam os critérios de inclusão da pesquisa. Na Biblioteca Virtual em Saúde, apenas um artigo foi eleito para análise. Dentre todos os artigos analisados, seis foram publicados entre 2010 e 2015 (Walker et al., 2011; McColloug, Small e Prady, 2013; Murphy et al., 2014; Harris et al., 2012; Nemec e LaMaster, 2014; Wimpeny et al., 2010) e três foram publicados entre 2010 e 2005 (Barrett et al., 2009; Barwick, Peters e Boydell, 2009; Huckson e Davies, 2007). A maioria dos estudos (quatro artigos) encontrados foi proveniente dos Estados Unidos da América (Barret et al., 2009; Nemec e LaMaster, 2014; Harris et al., 2012; Walker et al., 2011); houve duas publicações do Reino Unido (McCollough, Small e Prady, 2013; Wimpenny et al., 2010), duas do Canadá (Barwick, Peters e Boydell, 2009; Murphy et al., 2014) e uma da Austrália (Huckson e Davies, 2007). Esses dados indicaram que a produção é recente, crescente e localiza-se, principalmente, em países de origem anglo-saxã.

A população-alvo mostrou-se bastante heterogênea: quatro pesquisas estiveram voltadas para profissionais de única especialidade (Barret et al., 2009; Harris et al., 2012; McCollough, Small e Prady, 2013; Wimpenny et al., 2010); duas trabalharam com equipes multidisciplinares (Barwick, Peters e Boydell, 2009; Huckson e Davies, 2007); uma com equipes multidisciplinares, pacientes e familiares (Walker et al., 2011); uma com profissionais de uma única especialidade, pacientes e familiares (Murphy et al., 2014); uma com educadores de nível superior (Nemec e LaMaster, 2014).

Em três estudos (Barret et al., 2009; Harris et al., 2012; Huckson e Davies, 2007), a comunidade de prática foi constituída com o objetivo de capacitar profissionais para o cuidado em saúde mental. Em outros três (Barwick, Peters e Boydell, 2009; Walker et al., 2011; Wimpenny et al., 2010), o objetivo central foi implementar um novo modelo ou ferramenta de trabalho; e em dois estudos (McCollough, Small e Prady, 2013; Murphy et al., 2014) buscou-se melhorar as práticas realizadas nos serviços. Um estudo teve o objetivo de capacitar educadores acadêmicos para o ensino de saúde mental (Nemec e LaMaster, 2014). 
Todos os estudos utilizaram-se da descrição e análise da experiência da CoP. Barret e colaboradores (2009) e Barwick, Peters e Boydell (2009) também utilizaram questionários como fonte de coleta de dados prévios à participação e para avaliar as mudanças na prática.

O Quadro 1 sintetiza as principais informações dos estudos avaliados.

\section{Quadro 1}

\begin{tabular}{|c|c|c|c|c|}
\hline \multicolumn{5}{|c|}{ Caracterização dos artigos selecionados na revisão sistemática } \\
\hline $\begin{array}{c}\text { Autor(es)/ } \\
\text { ano }\end{array}$ & Título & Revista & Objetivo & Resultados \\
\hline $\begin{array}{l}\text { Walker et } \\
\text { al., } 2011\end{array}$ & $\begin{array}{l}\text { The National } \\
\text { Wraparound } \\
\text { Initiative: a } \\
\text { community of } \\
\text { practice approach } \\
\text { building } \\
\text { knowledge in the } \\
\text { field of children's } \\
\text { mental health }\end{array}$ & $\begin{array}{l}\text { Best Practices } \\
\text { in Mental } \\
\text { Health }\end{array}$ & $\begin{array}{l}\text { Descrever a experiência } \\
\text { de profissionais de } \\
\text { serviços de saúde } \\
\text { mental infantil, } \\
\text { pacientes e familiares } \\
\text { que constituíram uma } \\
\text { CoP para sistematizar o } \\
\text { modelo de prática que } \\
\text { utilizam. }\end{array}$ & $\begin{array}{l}\text { A despeito de muitos desafios } \\
\text { e tensões, a experiência sugere } \\
\text { que esse tipo de abordagem } \\
\text { colaborativa pode ser usado } \\
\text { como um meio para soluções } \\
\text { criativas de problemas nos } \\
\text { serviços. }\end{array}$ \\
\hline $\begin{array}{l}\text { McCollough, } \\
\text { Small e } \\
\text { Prady, } 2013\end{array}$ & $\begin{array}{l}\text { Improving } \\
\text { smoking } \\
\text { cessation data } \\
\text { collection via } \\
\text { a health visitor } \\
\text { Community of } \\
\text { Practice }\end{array}$ & $\begin{array}{l}\text { Community } \\
\text { Practitioner }\end{array}$ & $\begin{array}{l}\text { Avaliar a qualidade de } \\
\text { dados sobre tabagismo } \\
\text { coletados por agentes } \\
\text { comunitários de } \\
\text { saúde e promover a } \\
\text { aproximação entre } \\
\text { pesquisadores e } \\
\text { profissionais na prática. }\end{array}$ & $\begin{array}{l}\text { A CoP potencializou a } \\
\text { identificação de problemas, } \\
\text { a construção coletiva e a } \\
\text { implementação de soluções, } \\
\text { com monitoramento por } \\
\text { período preestabelecido. }\end{array}$ \\
\hline $\begin{array}{l}\text { Murphy et } \\
\text { al., } 2014\end{array}$ & $\begin{array}{l}\text { A theory- } \\
\text { informed } \\
\text { approach to } \\
\text { mental health } \\
\text { care capacity } \\
\text { building for } \\
\text { pharmacists }\end{array}$ & $\begin{array}{l}\text { International } \\
\text { Journal of } \\
\text { Mental Health } \\
\text { Systems }\end{array}$ & $\begin{array}{l}\text { Descrever o } \\
\text { desenvolvimento e } \\
\text { a implementação } \\
\text { de programa de } \\
\text { capacitação para } \\
\text { farmacêuticos atuarem } \\
\text { com pessoas com } \\
\text { adoecimento mental, } \\
\text { em uma abordagem } \\
\text { comunitária e territorial. }\end{array}$ & $\begin{array}{l}\text { As teorias e os referenciais } \\
\text { teóricos utilizados, assim } \\
\text { como as experiências } \\
\text { práticas, propiciaram maior } \\
\text { apropriação de conhecimentos } \\
\text { aos farmacêuticos, estando } \\
\text { inseridos em serviços de } \\
\text { abordagem territorial, para a } \\
\text { oferta de serviços a pessoas que } \\
\text { viveram adoecimento mental. }\end{array}$ \\
\hline
\end{tabular}


Continuação - Quadro 1

\begin{tabular}{|c|c|c|c|c|}
\hline \multicolumn{5}{|c|}{ Caracterização dos artigos selecionados na revisão sistemática } \\
\hline $\begin{array}{c}\text { Autor(es)/ } \\
\text { ano }\end{array}$ & Título & Revista & Objetivo & Resultados \\
\hline $\begin{array}{l}\text { Harris et al., } \\
2012\end{array}$ & $\begin{array}{l}\text { Communities of } \\
\text { Practice: creating } \\
\text { the Bilingual } \\
\text { School Mental } \\
\text { Health Network } \\
\text { in Colorado }\end{array}$ & Communiqué & $\begin{array}{l}\text { Descrever uma } \\
\text { CoP composta por } \\
\text { profissionais atuantes } \\
\text { em escolas do estado } \\
\text { do Colorado para } \\
\text { melhorar os serviços } \\
\text { prestados à clientela } \\
\text { com base na melhor } \\
\text { compreensão de } \\
\text { aspectos culturais e } \\
\text { linguísticos. }\end{array}$ & $\begin{array}{l}\text { A CoP foi considerada uma } \\
\text { estratégia inovadora para o } \\
\text { avanço da prática educacional } \\
\text { para trabalhar de maneira } \\
\text { colaborativa, representando um } \\
\text { poderoso meio de compartilhar } \\
\text { recursos e conhecimento, } \\
\text { especialmente com orçamentos } \\
\text { reduzidos. }\end{array}$ \\
\hline $\begin{array}{l}\text { Wimpenny } \\
\text { et al., } 2010\end{array}$ & $\begin{array}{l}\text { Implementing } \\
\text { the Model } \\
\text { of Human } \\
\text { Occupation } \\
\text { across a } \\
\text { mental health } \\
\text { occupational } \\
\text { therapy service: } \\
\text { Communities } \\
\text { of Practice and } \\
\text { a participatory } \\
\text { change process }\end{array}$ & $\begin{array}{l}\text { British } \\
\text { Journal of } \\
\text { Occupational } \\
\text { Therapy }\end{array}$ & $\begin{array}{l}\text { Descrever uma } \\
\text { pesquisa-ação } \\
\text { que investigou a } \\
\text { implementação do } \\
\text { modelo de ocupação } \\
\text { humana dentro de } \\
\text { um serviço de terapia } \\
\text { ocupacional em saúde } \\
\text { mental. }\end{array}$ & $\begin{array}{l}\text { Os resultados enfatizaram } \\
\text { a importância do } \\
\text { desenvolvimento de um local } \\
\text { para aprendizagem crítica. } \\
\text { Identificaram-se barreiras para } \\
\text { a implementação da teoria, } \\
\text { superadas por esforço coletivo } \\
\text { numa dialética compartilhada. } \\
\text { O sucesso do desenvolvimento } \\
\text { da CoP requereu considerações } \\
\text { cautelosas de diversas } \\
\text { influências interconectadas, } \\
\text { incluindo questões pessoais, } \\
\text { relacionais e contextuais. }\end{array}$ \\
\hline $\begin{array}{l}\text { Barrett et } \\
\text { al., } 2009\end{array}$ & $\begin{array}{l}\text { Nurses are the } \\
\text { key to improving } \\
\text { mental health } \\
\text { services in } \\
\text { low- and } \\
\text { middle-income } \\
\text { countries }\end{array}$ & $\begin{array}{l}\text { International } \\
\text { Nursing Review }\end{array}$ & $\begin{array}{l}\text { Apresentar os } \\
\text { resultados de } \\
\text { uma pesquisa que } \\
\text { foi realizada em } \\
\text { setembro de 2007, } \\
\text { encomendada pela } \\
\text { Organização Mundial } \\
\text { da Saúde (OMS) } \\
\text { e pelo Conselho } \\
\text { Internacional de } \\
\text { Enfermagem. }\end{array}$ & $\begin{array}{l}\text { Tanto as respostas do } \\
\text { questionário quanto as } \\
\text { discussões do fórum on-line } \\
\text { demonstraram que há interesse } \\
\text { em melhorar as práticas de } \\
\text { saúde mental nos serviços } \\
\text { prestados por enfermeiras em } \\
\text { países subdesenvolvidos e em } \\
\text { desenvolvimento. Ressalta-se } \\
\text { que há necessidade de ampliar } \\
\text { o número de enfermeiras } \\
\text { com especialização em saúde } \\
\text { mental e formação mínima em } \\
\text { saúde mental em todo curso de } \\
\text { enfermagem. }\end{array}$ \\
\hline
\end{tabular}

continua> 
Continuação - Quadro 1

\begin{tabular}{|c|c|c|c|c|}
\hline $\begin{array}{c}\text { Autor(es)/ } \\
\text { ano }\end{array}$ & Título & Revista & Objetivo & Resultados \\
\hline $\begin{array}{l}\text { Barwick, } \\
\text { Peters e } \\
\text { Boydell, } \\
2009\end{array}$ & $\begin{array}{l}\text { Getting to } \\
\text { uptake: do } \\
\text { Communities } \\
\text { of Practice } \\
\text { support the } \\
\text { implementation } \\
\text { of Evidence- } \\
\text { Based Practice? }\end{array}$ & $\begin{array}{l}\text { Journal of } \\
\text { the Canadian } \\
\text { Academy of } \\
\text { Child and } \\
\text { Adolescent } \\
\text { Psychiatry }\end{array}$ & $\begin{array}{l}\text { Examinar os } \\
\text { benefícios de uma } \\
\text { CoP no contexto } \\
\text { da saúde mental } \\
\text { infantil de Ontário, } \\
\text { Canadá, a partir da } \\
\text { adoção obrigatória } \\
\text { de um instrumento } \\
\text { padronizado para } \\
\text { medir resultados das } \\
\text { intervenções (palestras } \\
\text { e CoP) e monitorar as } \\
\text { respostas dos clientes } \\
\text { ao tratamento. }\end{array}$ & $\begin{array}{l}\text { Os grupos não se mostraram } \\
\text { diferentes no que se refere à } \\
\text { disposição para mudanças e não } \\
\text { reportaram mudanças na prática. } \\
\text { Entretanto, os participantes da CoP } \\
\text { mostraram melhor compreensão } \\
\text { da ferramenta na prática e de } \\
\text { seu conteúdo; estavam mais } \\
\text { satisfeitos com esse tipo de } \\
\text { suporte oferecido do que aqueles } \\
\text { participantes que estiveram } \\
\text { inseridos nas práticas usuais de } \\
\text { implementação da ferramenta } \\
\text { (como palestras e suporte on-line). }\end{array}$ \\
\hline $\begin{array}{l}\text { Huckson } \\
\text { e Davies, } \\
2007\end{array}$ & $\begin{array}{l}\text { Closing evidence } \\
\text { to practice gaps } \\
\text { in Emergency } \\
\text { Care: the } \\
\text { Australian } \\
\text { experience }\end{array}$ & $\begin{array}{l}\text { Academic } \\
\text { Emergency } \\
\text { Medicine }\end{array}$ & $\begin{array}{l}\text { Descrever o uso da CoP } \\
\text { para oferecer suporte } \\
\text { a profissionais que } \\
\text { trabalham em serviços } \\
\text { de emergência para } \\
\text { implementar prática } \\
\text { baseada em evidência. }\end{array}$ & $\begin{array}{l}\text { Por meio da CoP, a comunidade } \\
\text { clínica elegeu prioridades } \\
\text { para o programa de cuidado } \\
\text { emergencial e mostrou- } \\
\text { se ativamente engajada } \\
\text { no desenvolvimento e na } \\
\text { implementação das iniciativas. }\end{array}$ \\
\hline $\begin{array}{l}\text { Nemec e } \\
\text { LaMaster, } \\
2014\end{array}$ & $\begin{array}{l}\text { Education and } \\
\text { training column: } \\
\text { Communities of } \\
\text { Practice }\end{array}$ & $\begin{array}{l}\text { Psychiatric } \\
\text { Rehabilitation } \\
\text { Journal }\end{array}$ & $\begin{array}{l}\text { Descrever os } \\
\text { componentes- } \\
\text { chave de uma } \\
\text { CoP denominada } \\
\text { Consortium } \\
\text { of Psychiatric } \\
\text { Rehabilitation } \\
\text { Educators (CPRE). }\end{array}$ & $\begin{array}{l}\text { O desenvolvimento da CoP } \\
\text { ofereceu a seus membros uma } \\
\text { chance de desenvolvimento } \\
\text { profissional, compartilhando } \\
\text { conhecimento sobre a } \\
\text { reabilitação psiquiátrica } \\
\text { e entrando em contato } \\
\text { com experts, e também a } \\
\text { oportunidade de desenvolver } \\
\text { melhor compreensão do } \\
\text { sistema geral de cuidado em } \\
\text { saúde mental, compartilhando } \\
\text { conhecimento por meio das } \\
\text { linhas organizacionais. }\end{array}$ \\
\hline
\end{tabular}

Fonte: As autoras.

Nota: CoP - Comunidade Prática.

\section{Potencialidades e desafios da CoP}

A CoP mostrou-se uma ferramenta capaz de contemplar objetivos de diferentes naturezas, pois - como Barwick, Peters e Boydell (2009) observam - ela faz com que o processo de formação se torne mais do que uma simples transmissão de conhecimento, 
daquele que sabe mais para aquele que sabe menos, potencializando um esforço que inclui as complexidades da prática e dos profissionais. Considera que a transmissão de conhecimento deve estar situada no contexto em que ele será usado e desenvolvido.

Especialmente no que se refere ao trabalho do campo da saúde mental com abordagem territorial, a aprendizagem e o próprio desempenho da prática em si não estão pautados apenas em evidências científicas, já que os estudos produzidos nessa área ainda são, geralmente, bastante voltados para o tratamento medicamentoso na perspectiva hospitalar (Thornicroft e Tansella, 2010). Assim, os profissionais necessitam também da experiência, para que sejam capazes de desempenhar suas funções dentro da equipe - sendo, inclusive, capazes de incluir familiares e outros membros da rede social de suporte do paciente ativamente no processo de planejamento e execução do tratamento. E quando o aprendizado dos profissionais consegue abarcar as peculiaridades de seu local de trabalho e se faz coerente à realidade, ele se torna, de fato, efetivo (Thornicroft e Tansella, 2010).

Walker e colaboradores (2011) elucidam a questão supracitada ao descreverem sua experiência em uma comunidade de prática composta por profissionais de diferentes áreas, usuários e seus familiares, na tentativa de formalizar o método de trabalho que preconiza o favorecimento à adesão ao tratamento e a obtenção de melhores resultados - utilizando-se da participação ativa de todos os integrantes da rede social de suporte em todas as etapas do cuidado. Nesse sentido, é possível traçar um paralelo com Murphy e colaboradores (2014), os quais, em sua experiência, envolveram pacientes que já experimentaram sofrimento psíquico em uma comunidade de prática que, aliada a outros instrumentos formativos, teve como objetivo treinar farmacêuticos para o cuidado de pessoas com transtornos mentais em serviços de abordagem territorial. A formação básica desses profissionais não é considerada suficientemente boa para que eles sejam capazes de manejar questões relativas à tomada de medicamentos no contato direto com os usuários dos serviços. Nesse sentido, a aproximação direta, por meio da comunidade de prática, com esses sujeitos mostrou-se de grande valor para o processo de aprendizagem.

Para o cuidado em saúde mental, diante da necessidade de invenção de práticas centradas no contexto dos sujeitos sob cuidado e das necessidades que se modificam constantemente, a integração, em uma mesma experiência formativa, dos diversos atores envolvidos no cuidado favorece a mudança das subjetividades envolvidas no processo (Macedo, Albuquerque e Medeiros, 2014; Onocko-Campos, 2009; Thornicroft e Tansella, 2010).

No Quadro 2, buscamos relacionar o formato em que cada comunidade de prática foi desenvolvida com o número de pessoas que participaram ativamente dela, assim como os tipos de ferramenta formativa escolhidos.

A maioria dos estudos mesclou atividades virtuais e presenciais. É possível notar que o uso de ferramentas virtuais se relaciona diretamente com um número maior de participantes, ainda que alguns autores não informem o número exato de participantes das comunidades de prática em seus estudos. 
Quadro 2

\begin{tabular}{|c|c|c|c|}
\hline Autor(es)/ano & Formato & Ferramentas formativas utilizadas & Número de participantes \\
\hline Walker et al., 2011 & $\begin{array}{l}\text { Virtual, com } \\
\text { eventos } \\
\text { presenciais }\end{array}$ & $\begin{array}{l}\text { Estratégias virtuais: e-mail, } \\
\text { questionários on-line e outros } \\
\text { processos mediados pela internet; } \\
\text { encontros anuais. }\end{array}$ & 200 \\
\hline $\begin{array}{l}\text { McColloug, Small e } \\
\text { Prady, } 2013\end{array}$ & Presencial & $\begin{array}{l}\text { Reuniões regulares com tempo } \\
\text { específico de duração. }\end{array}$ & Não é especificado \\
\hline Murphy et al., 2014 & $\begin{array}{l}\text { Virtual (grupo } \\
\text { menor), } \\
\text { seguido de } \\
\text { formação } \\
\text { presencial }\end{array}$ & $\begin{array}{l}\text { Website com comunicações regulares, } \\
\text { jornal e mídias sociais. }\end{array}$ & $\begin{array}{l}210 \\
\text { (35 por meio virtual; cada um } \\
\text { destes formou outros cinco } \\
\text { grupos presencialmente) }\end{array}$ \\
\hline Harris et al., 2012 & $\begin{array}{l}\text { Presencial, } \\
\text { com recursos } \\
\text { virtuais }\end{array}$ & $\begin{array}{l}\text { Reuniões que poderiam também ser } \\
\text { assistidas via videoconferência por } \\
\text { aqueles que não conseguiam se deslocar. }\end{array}$ & 85 \\
\hline $\begin{array}{l}\text { Wimpenny et al., } \\
2010\end{array}$ & Presencial & $\begin{array}{l}\text { Reuniões mensais e supervisões } \\
\text { individuais esporádicas com } \\
\text { profissionais de outras especialidades. }\end{array}$ & 15 \\
\hline Barrett et al., 2009 & Virtual & $\begin{array}{l}\text { Fórum on-line com quatro subgrupos } \\
\text { de discussão com diferentes temas. }\end{array}$ & 615 (80 países) \\
\hline $\begin{array}{l}\text { Barwick, Peters e } \\
\text { Boydell, } 2009\end{array}$ & Presencial & Reuniões periódicas. & 18 \\
\hline $\begin{array}{l}\text { Huckson e Davies, } \\
2007\end{array}$ & $\begin{array}{l}\text { Predominantemente } \\
\text { virtual, com } \\
\text { oportunidades } \\
\text { de contato } \\
\text { presencial }\end{array}$ & $\begin{array}{l}\text { Website, com a elaboração de manuais } \\
\text { e outros produtos das discussões que, } \\
\text { em seguida, eram disponibilizados } \\
\text { para download; o artigo relata outras } \\
\text { oportunidades para comunicação } \\
\text { interpessoal que não são descritas. }\end{array}$ & Não é especificado \\
\hline $\begin{array}{l}\text { Nemec e LaMaster, } \\
2014\end{array}$ & $\begin{array}{l}\text { Presencial, } \\
\text { com uso } \\
\text { pouco eficaz } \\
\text { de recursos } \\
\text { virtuais }\end{array}$ & $\begin{array}{l}\text { Estudo de textos; palestras com } \\
\text { experts; página no Facebook; site com } \\
\text { área restrita, protegida por senha, para } \\
\text { acesso de membros. }\end{array}$ & Não é especificado \\
\hline
\end{tabular}

Fonte: As autoras. 
Barwick, Peters e Boydell (2009) e Barrett e colaboradores (2009) ressaltam que uma das grandes potencialidades que puderam encontrar no uso da comunidade de prática foi sua capacidade de transcender limites geográficos, podendo desenvolver-se completamente em ambientes virtuais (sites, e-mail, redes sociais e fóruns on-line). Ainda que majoritariamente a CoP esteja pautada em encontros presenciais, participantes que se encontram distantes podem utilizar ferramentas como videoconferência (Harris et al., 2012), possibilitando, por exemplo, que enfermeiros de países de baixa e média renda - que muitas vezes atuam em locais isolados e de grande vulnerabilidade - recebam capacitação para o cuidado de pacientes em sofrimento psíquico a partir do contato com profissionais experientes (Barrett et al., 2009). Harris e colaboradores (2012) e Barrett e colaboradores (2009) também consideram que essa ferramenta é capaz de transcender limites organizacionais, pois podem unir, em um mesmo grupo, pessoas que realizam diferentes funções dentro de um serviço, ultrapassando inclusive barreiras hierárquicas.

A superação de limites geográficos, organizacionais e hierárquicos apresenta-se como importante característica da ferramenta da CoP, indo ao encontro da superação de complexidades da formação permanente em saúde e saúde mental (Macedo, Albuquerque e Medeiros, 2014).

Os desafios enfrentados foram indicados por diversos autores (Barwick, Peters e Boydell, 2009; McCollough, Small e Prady, 2013; Nemec e LaMaster, 2014; Walker et al., 2011; Wimpenny et al., 2010). Um ponto em comum abarca a questão do investimento financeiro. Walker e colaboradores (2011), Nemec e LaMaster (2014) e Barwick e colaboradores (2009) observam que o desenvolvimento das comunidades de prática esbarra na falta ou na precariedade de investimento, bem como na impossibilidade de o grupo produzir material com valor financeiro capaz de sustentar a continuidade do processo de modo independente (Walker et al., 2011). Isso interferiu diretamente na remuneração de organizadores e coordenadores das comunidades de prática, na contratação de palestrantes (Nemec e LaMaster, 2014; Walker et al., 2011) e na possibilidade de os profissionais se ausentarem do trabalho para participar dos encontros presenciais (Barwick, Peters e Boydell, 2009).

Nemec e LaMaster (2014) e Walker e colaboradores (2011) ressaltam que o financiamento é um elemento fundamental para dar suporte às atividades da comunidade de prática e à documentação do conhecimento gerado, e que esse foi um dos maiores desafios enfrentados - realidade presente em várias atividades relacionadas ao campo da saúde mental (Organização Mundial da Saúde, 2005; Thornicroft e Tansella, 2010). Além disso, Walker e colaboradores (2011) destacam que a produção de conhecimento é financiada por fundos de pesquisa, cujas expectativas tensionam-se diante da abordagem da comunidade de prática, que é, por natureza, imprevisível e incontrolável. 
Outra dificuldade relatada são os conflitos grupais de diferentes naturezas que ocorreram dentro das comunidades de prática, indicando que o manejo de tais conflitos deverá ser voltado para a manutenção da produtividade do grupo, devendo-se tornar força motriz para a produção de conhecimento. A emergência de conflitos pode decorrer de fatores como a insatisfação com os temas discutidos dentro da CoP ou com os referenciais eleitos para esses temas, ou ainda a mobilização de estruturas pessoais de defesa contra uma proposta de mudança na prática ou outros movimentos do grupo que possam ser compreendidos como ataque pessoal (Walker et al., 2011; Wimpenny et al., 2010).

Em direção similar, a descontinuidade entre o que é discutido e compreendido com clareza dentro da comunidade de prática e as características inerentes ao campo em que ela se dá transforma-se, muitas vezes, em barreiras para a aplicação dos conhecimentos adquiridos naquele ambiente de formação (Wimpenny et al., 2010), ou para tornar aquilo que é discutido pelo grupo de modo dinâmico em um produto concreto (Walker et al., 2011). Wenger (1998) discute essa característica como uma tensão entre a participação das pessoas e a reificação dessa participação em produtos que possam servir como a materialidade da aprendizagem e, ao mesmo tempo, possibilitem análises e reflexões, pois esse produto pode não estar alinhado com o que é almejado pela CoP.

Ainda na ótica dos conflitos grupais, outro desafio descrito por Walker e colaboradores (2011) foi o manejo da tensão entre as atividades designadas para serem trabalhadas no encontro e as atividades emergentes (Wenger, 1998). O manejo dessa tensão é crucial para a manutenção da produtividade do grupo, pois não se sentir contemplado pelas decisões tomadas pode favorecer o dispersar-se e gerar o não cumprimento das tarefas estabelecidas, bem como levar os membros ao estado de insatisfação supracitado (Marcolino, Lourenço e Reali (2017).

Marcolino e Reali (2012) destacam a necessidade do manejo de conflitos em comunidades de prática, de modo que aspectos implícitos possam se tornar explícitos para o grupo e serem assim alvo de reflexões e potenciais elementos de transformações. Além disso, ressaltam o quanto o coordenador precisa ter habilidades flexíveis de manejo do grupo e do trabalho no aqui-e-agora, explicitando combinados prévios, explicações, tensões emergentes, compreensões diferentes, de modo a ajudar a $\mathrm{CoP}$ a sair de qualquer paralisia produtiva.

\section{Considerações finais}

O cuidado em saúde mental é complexo, de natureza multidisciplinar e majoritariamente dependente de recursos humanos, fazendo uso restrito de tecnologias de outras naturezas. Nesse sentido, o desenvolvimento profissional torna-se uma área de preocupação e investimento para o campo. Em nossa revisão de literatu- 
ra, a comunidade de prática mostrou-se um referencial teórico e metodológico interessante e efetivo para fomentar mudanças na prática, em seus aspectos cognitivos e subjetivos, em diversos contextos de prática, objetivos e abrangência. Entretanto, o sucesso dessa iniciativa depende de muitos fatores, principalmente de financiamento adequado e capacidade de manejo de tensões grupais.

Os estudos são recentes e indicam uma perspectiva internacional de produção de conhecimento utilizando-se a comunidade de prática para formação e desenvolvimento de profissionais em seus contextos reais de trabalho. No Brasil, há trabalhos utilizando a comunidade de prática como referencial teórico-metodológico (El-Hani e Greca, 2011; Binotto et al., 2007; Silva, 2010), o que indica possibilidades de adequação cultural, embora não no campo da saúde mental.

Diante do desafio da melhoria de formação e atuação para o cuidado em saúde e saúde mental no Brasil, esperamos que esta revisão possa elucidar algumas possibilidades para a criação de estratégias formativas e investigativas nessa direção.

\section{Colaboradores}

Todas as autoras participaram da redação do manuscrito. A primeira autora foi responsável pela elaboração da análise e discussão dos dados. A segunda e a terceira autoras contribuíram também para a discussão dos dados e a revisão do texto. Informamos que este trabalho não possui conflitos de interesses.

Resumen La atención en salud mental depende de recursos humanos calificados para responder dinámicamente a necesidades de los usuarios del servicio. Esta calificación por la reflexión sobre los procesos de trabajo es esencial para un cambio efectivo a una perspectiva comunitaria y biopsicosocial. La Comunidad de Práctica es un marco teórico que fomenta el aprendizaje colaborativo a través del compromiso mutuo en proyectos comunes y negociación de significados. La investigación objetivó sistematizar el uso de la Comunidad de Práctica para mejorar la atención en salud mental, mediante revisión sistemática en Portal de Periódicos Capes y Biblioteca Virtual en Salud en marzo de 2015. Encontramos diecinueve artículos y nueve fueron seleccionados para análisis. Los estudios evaluaron la Comunidad de Práctica como positiva para mejorar los servicios ofrecidos a la población, proporcionar formación con expertos, y poner en práctica nuevos métodos de trabajo. Destacanse puntos débiles (recursos financieros, desalineación inicial entre los participantes, conflictos de grupo) y potencialidades (compromiso real de los participantes, participación virtual, acceso a especialistas, integración de profesionales, usuarios y familias en un solo proyecto; necesidades de formación contextuales). Esperamos aclarar posibilidades para estrategias de formación y de investigación para la educación permanente en salud mental.

Palabras clave salud mental; educación continua; comunidad de práctica; recursos humanos en salud; práctica profesional. 


\section{Notas}

\footnotetext{
${ }^{1}$ Universidade Federal de São Carlos, Departamento de Terapia Ocupacional, São Carlos, São Paulo, Brasil. <taisquevedo@gmail.com>

Correspondência: Universidade Federal de São Carlos, Rodovia Washington Luís, km 235, CEP 13565-905, São Carlos, São Paulo, Brasil.

${ }^{2}$ Universidade Estadual de Campinas, Residência Multiprofissinal em Saúde Mental e Saúde Coletiva, Campinas, São Paulo, Brasil.

$<$ elianenfantinatti@gmail.com>

${ }^{3}$ Universidade Federal de São Carlos, Departamento de Terapia Ocupacional, São Carlos, São Paulo, Brasil.

<alanafornereto@gmail.com>
}

\section{Referências}

BARDIN, Laurence. Análise de conteúdo. Lisboa: Edições 70, 2011.

BARRETT, Thomas et al. Nurses are the key to improving mental health services in low- and middle-income countries. International Nursing Review, Geneva, v. 56, n. 1, p. 138-141, mar. 2009.

BARWICK, Melanie A.; PETERS, Julia; BOYDELL, Katherine. Getting to uptake: do Communities of Practice support the implementation of Evidence-Based Practice? Journal of the Canadian Academy of Child and Adolescent Psychiatry, Ottawa, v. 18, n. 1, p. 16-29, fev. 2009.

BINOTTO, Erlaine et al. A comunidade de prática como ferramenta da criação de conhecimento no contexto do agronegócio. Desenvolvimento em Questão, Brasília, v. 5, n. 10, p. 11-42, 2007.

BRASIL. Ministério da Saúde. Portaria n. 278, de 27 de fevereiro de 2014. Institui a Política Nacional de Educação Permanente em Saúde como estratégia do Sistema Único de Saúde para a formação e o desenvolvimento de trabalhadores para o setor. Disponível em: $<$ http://bvsms.saude.gov.br/bvs/saudelegis/ gm/2014/prt0278_27_02_2014.html>. Acesso em: 20 out. 2015.

BRASIL. Ministério da Saúde. Portaria n. 1.996, de 20 de agosto de 2007. Estabelece as diretrizes para a implementação da Política Nacional de Educação Permanente em Saúde. Disponível em: <http://www.saude.mt.gov. br/upload/legislacao/1996-\%5B2968-120110SES-MT\%5D.pdf>. Acesso em: 20 out. 2015.

CASTRO, Cristiane P.; CAMPOS, Gastão W. S. Apoio institucional Paideia como estratégia para educação permanente em saúde. Trabalho, Educação e Saúde, Rio de Janeiro, v. 12, n. 1, p. 29-50, 2014. Disponível em: $<$ http://www.scielo.br/scielo.php?script=sci arttext\&pid=S1981-77462014000100003\&lng $=$ pt\&nrm $=$ iso $>$. Acesso em: 14 set. 2016 .

CUBAS, Márcia R. et al. Componentes da teoria social de aprendizagem numa ferramenta para ensino na enfermagem. Revista Brasileira de Enfermagem, Brasília, v. 68, n. 5, p. 906-912, 2015. Disponível em: <http://www.scielo.br/ scielo.php?script $=$ sci_arttext $\&$ pid $=$ S0034$71672015000500906 \& \operatorname{lng}=\mathrm{en} \& \mathrm{nrm}=\mathrm{iso}>$. Acesso em: 4 ago. 2016.

DAVINI, María C. Educación permanente en salud. Washington: OPS, 1995. Serie PALTEX para Ejecutores de Programas de Salud, n. 38. Disponível em: <http://iris.paho.org/ 
xmlui/bitstream/handle/123456789/3104/ Educaci $\%$ F3n \%20permanente $\% 20$ en $\% 20$ salud.pdf?sequence $=1>$. Acesso em: 17 jan. 2015.

EL-HANI, Charbel N.; GRECA, Ileana M. Participação em uma comunidade virtual de prática desenhada como meio de diminuir a lacuna pesquisa-prática na educação em biologia. Ciência \& Educação, Bauru, v. 17, n. 3, p. 579-601, 2011.

FABBRI, Sandra et al. Improvements in the Start tool to better support the systematic review process. In: PROCEEDINGS OF THE 20TH INTERNATIONAL CONFERENCE ON EVALUATION AND ASSESSMENT IN SOFTWARE ENGINEERING (EASE'16), Limerick, Ireland, jun. 2016. Disponível em: https:// dl.acm.org/citation.cfm?id=2916013. Acesso em: 20 jul. 2015.

FLORES, Giovana E.; OLIVEIRA, Dora L. L.; ZOCCHE, Denise A. A. Educação permanente no contexto hospitalar: a experiência que ressignifica o cuidado em enfermagem. Trabalho, Educação e Saúde, Rio de Janeiro, v. 14, n. 2, p. 487-504, 2016. Disponível em $<$ http://www.scielo.br/scielo.php?script=sci_ arttext\&pid $=$ S1981-77462016000200487\&lng $=$ pt\&nrm $=$ iso $>$. Acesso em: 14 set. 2016 .

FRANCO, Túlio B. Produção do cuidado e produção pedagógica: integração de cenários do Sistema Único de Saúde no Brasil. Interface: Comunicação, Saúde e Educação, Botucatu, v. 11, n. 23, p. 427-438, set.-dez. 2007.

GALHEIGO, Sandra M. et al. Exchanging knowledge within a community of practice: toward an epistemology of practice in Occupational Therapy paediatric hospital care. Cadernos Brasileiros de Terapia Ocupacional, São Carlos, v. 25, n. 3, p. 449-459, 2017. Disponível em: <https://doi.org/10.4322/25268910.ctoAO1119>. Acesso em: 24 jul. 2017.

HARRIS, Bryn et al. Communities of Practice: creating the Bilingual School Mental Health Network in Colorado. Communiqué, Washington, v. 40, n. 6, p. 22-23, 2012.
HUCKSON, Susan; DAVIES, Jan. Closing evidence to practice gaps in Emergency Care: the Australian experience. Academic Emergency Medicine, Malden, v. 14, n. 11, p. 1.058-1.063, nov. 2007

LIMA, Elizabeth M. F. A.; GHIRARDI, Maria I. G. Transdisciplinaridade e práticas híbridas em saúde mental. Revista de Terapia Ocupacional da Universidade de São Paulo, São Paulo, v. 19, n. 3, p. 153-158, set.-dez. 2008.

LINDE, Klaus; WILLICH, Stefan N. How objective are systematic reviews? Differences between reviews in complementary medicine. Journal of Royal Society Medicine, Londres, v. 96, n. 1, p. 17-22, 2003.

MACEDO, Neusa B.; ALBUQUERQUE, Paulette C.; MEDEIROS, Kátia R. O desafio da implementação da educação permanente na gestão da educação na saúde. Trabalho, Educação e Saúde [online], Rio de Janeiro, v. 12, n. 2, p. 379-401, 2014. Disponível em: <http://dx.doi. org/10.1590/S1981-77462014000200010>. Acesso em: 20 jul. 2015.

MARCOLINO, Taís Q; LOURENÇO, Gerusa F.; REALI, Aline M. M. R. 'Isso eu levo para a vida!': aprendizagem da prática profissional em uma comunidade de prática. Interface: Comunicação, Saúde e Educação, Botucatu, v. 17, n. 61, p. 411-420, 2017.

MARCOLINO, Taís Q.; REALI, Aline M. M. R. Rotas dissonantes e comunidade profissional: pistas para promover a aprendizagem colaborativa. In: REALI, Aline M. M. R. (org.). Desenvolvimento profissional da docência: teorias e práticas. São Carlos: EdUFSCar, 2012, p. 281-298.

McCULLOUGH, Bunny; SMALL, Neil; PRADY, Sthephani L. Improving smoking cessation data collection via a health visitor Community of Practice. Community Practitioner, Londres, v. 86, n. 5, p. 22-25, maio 2013.

MURPHY, Andrea L. et al. A theory-informed approach to mental health care capacity building for pharmacists. International Journal of 
Mental Health Systems, Melbourne, v. 8, n. 46, nov. 2014. Disponível em: <https://www. ncbi.nlm.nih.gov/pmc/articles/PMC4254206/>. Acesso em: 20 jul. 2015

NEMEC, Patricia B.; LaMASTER, Stephen Education and training column: Communities of Practice. Psychiatric Rehabilitation Journal, Washington, v. 37, n. 4, p. 336-338, dez. 2014.

ONOCKO-CAMPOS, Rosana T. et al. Avaliação da rede de centros de atenção psicossocial: entre a saúde coletiva e a saúde mental. Revista de Saúde Pública, São Paulo, v. 43, supl. 1, p. 16-22, 2009. Disponível em: <http://dx.doi. org/10.1590/S0034-89102009000800004>. Acesso em: jul. 2015.

ORGANIZAÇÃO MUNDIAL DA SAÚDE (OMS). Livro de recursos da OMS sobre saúde mental, direitos e legislação: cuidar, sim - excluir, não. Genebra: Organização Mundial da Saúde, 2005. Disponível em: $<$ http://www.who.int/mental_health/policy/ Livroderecursosrevisao_FINAL.pdf $>$. Acesso em: 20 jul. 2015

RAMOS, Wanusa R.; MANRIQUE, Ana L. Comunidade de prática de professores que ensinam matemática como espaço de negociações de significados sobre a resolução de problemas. Bolema: Boletim de Educação Matemática, Rio Claro, v. 29, n. 53, p. 979997, 2015. Disponível em: <http://www.scielo. br/scielo.php?script=sci_arttext\&pid $=$ S0103636X2015000300979\&lng=en \&nrm=iso > . Acesso em: 4 ago. 2016.
SILVA, Heloísa. Uma caracterização do Centro de Educação Matemática-CEM (1984-1997) como uma comunidade de prática de formação continuada de professores de matemática. Bolema: Boletim de Educação Matemática, Rio Claro, v. 23, n. 35 , p. 185-218, 2010. Disponível em: <http://hdl. handle.net/11449/71644> . Acesso em: 4 ago. 2016.

THORNICROFT, Graham; TANSELLA, Michele. Boas práticas em saúde mental comunitária. Barueri: Manole, 2010.

WALKER, Janet S. et al. The National Wraparound Initiative: a Community of Practice approach to building knowledge in the field of Children's Mental Health. Best Practices in Mental Health, Chicago, v. 7, n. 1, p. 26-45, jan. 2011.

WELCH, Amanda; DAWSON, Pamela. Closing the gap: collaborative learning as a strategy to embed evidence within occupational therapy practice. Journal of Evaluation in Clinical Practice, Medford, v. 12, n. 2, p. 227-238, 2006.

WENGER, Ethiènne. Communities of practice learning, meaning and identity. Cambridge: Cambridge University Press, 1998.

WIMPENNY, Katherine et al. Implementing the Model of Human Occupation across a mental health occupational therapy service: communities of practice and a participatory change process. British Journal of Occupational Therapy, Londres, v. 73, n. 11, p. 507-516, nov. 2010.

Recebido em 14/09/2016. Aprovado em 30/03/2017. 